\title{
A New Distance Measure for Trapezoidal Fuzzy Numbers
}

\author{
M. J. Ebadi, ${ }^{1}$ M. Suleiman, ${ }^{2}$ Fudziah Bt. Ismail, ${ }^{3}$ A. Ahmadian, ${ }^{2}$ \\ M. R. Balooch Shahryari, ${ }^{4}$ and S. Salahshour ${ }^{5}$ \\ ${ }^{1}$ Faculty of Marine Science, Chabahar Maritime University, Chabahar, Iran \\ ${ }^{2}$ Institute for Mathematical Research, Universiti Putra Malaysia, 43400 Serdang, Selangor, Malaysia \\ ${ }^{3}$ Department of Mathematics, Universiti Putra Malaysia, 43400 Serdang, Selangor, Malaysia \\ ${ }^{4}$ Department of Mathematics, Islamic Azad University, Kerman Branch, Kerman, Iran \\ ${ }^{5}$ Young Researchers and Elite Club, Islamic Azad University, Mobarakeh Branch, Mobarakeh, Iran
}

Correspondence should be addressed to S. Salahshour; soheilsalahshour@yahoo.com

Received 6 February 2013; Accepted 25 March 2013

Academic Editor: Tofigh Allahviranloo

Copyright (C) 2013 M. J. Ebadi et al. This is an open access article distributed under the Creative Commons Attribution License, which permits unrestricted use, distribution, and reproduction in any medium, provided the original work is properly cited.

We propose a new distance measure for the space of all trapezoidal fuzzy numbers using centroid point and left/right spread of trapezoidal fuzzy numbers. Moreover, the metric properties of suggested distance measure are investigated. Indeed, we show that for two arbitrary trapezoidal fuzzy numbers if the distance between centroid points and also the distance between left spreads and right spreads go to zero, then two given fuzzy numbers are equal. Consequently, we complete discussion about the relation between fuzzy number and its centroid which is the firstly discussed by Hadi-Vencheh and Allame (2010). To the best of our knowledge, this is first time in the literature that such metric is applied by centroid point.

\section{Introduction}

Fuzzy set theory [1] has been applied to many areas which need to manage uncertain and vague data. Such areas include approximate reasoning, decision making, optimization, and control, where ranking of fuzzy numbers is an important component of the decision process $[2,3]$.

There are several metrics to state the distance between fuzzy numbers. However, most of them have used the parametric form of fuzzy numbers or membership functions of given fuzzy numbers ([4-6] and references therein). But, in this paper we adopt a new approach to build a distance measure. To do so, we applied COG point to construct such distance measure. Before we proceed, we state some historical point of view about it.

Recently, Hadi-Vencheh and Allame [7] have suggested a relation between trapezoidal fuzzy numbers and their centroid formulas. To this end, they have solved the related linear system to characterize the components of each of the trapezoidal fuzzy numbers explicitly. However, they did not investigate the uniqueness of problem in this system. In other words, if the centroids and left/right spreads of two arbitrary trapezoidal fuzzy numbers are equal, do we conclude that these fuzzy numbers are identical?

To answer this essential question, we proposed a new distance measure for the space of trapezoidal fuzzy numbers, and metric properties of it are also stated.

The paper is organized as follows. In Section 2, we state some basic concepts. In Section 3, the centroid formula for trapezoidal fuzzy numbers is stated and also some required expressions are recalled using Hadi-Vencheh and Allame's paper [7]. In Section 4, we propose our new distance measure using centroid formula and left/right spreads. Moreover, the metric properties of proposed distance are discussed. Finally, the paper ends with conclusion.

\section{Basic Concepts}

In this section, some basic concepts are given. 
In the fuzzy theory, triangular and trapezoidal fuzzy numbers are used extensively [3], whose membership functions are, respectively, defined by

$$
\begin{gathered}
\mu_{A}(x)= \begin{cases}\left(\frac{x-a_{1}}{a_{2}-a_{1}}\right), & a_{1} \leq x \leq a_{2}, \\
\left(\frac{a_{3}-x}{a_{3}-a_{2}}\right), & a_{2} \leq x \leq a_{3}, \\
0, & \text { otherwise, }\end{cases} \\
\mu_{B}(x)= \begin{cases}\left(\frac{x-a_{1}}{a_{2}-a_{1}}\right), & a_{1} \leq x \leq a_{2} \\
1, & a_{2} \leq x \leq a_{3}, \\
\left(\frac{a_{4}-x}{a_{4}-a_{3}}\right), & a_{3} \leq x \leq a_{4}, \\
0, & \text { otherwise. }\end{cases}
\end{gathered}
$$

For brevity, triangular and trapezoidal fuzzy numbers are often denoted as $A=\left(a_{1}, a_{2}, a_{3}\right)$ and $B=\left(a_{1}, a_{2}, a_{3}, a_{4}\right)$. Clearly, triangular fuzzy numbers are special cases of trapezoidal fuzzy numbers with $a_{2}=a_{3}$.

An equivalent parametric definition is also given in [8] as follows.

Definition 1. A fuzzy number $u$ in parametric form is a pair $(\underline{u}, \bar{u})$ of functions $\underline{u}(r), \bar{u}(r), 0 \leq r \leq 1$, which satisfy the following requirements:

(1) $\underline{u}(r)$ is a bounded nondecreasing left continuous function in $(0,1]$ and right continuous at 0 ;

(2) $\bar{u}(r)$ is a bounded nonincreasing left continuous function in $(0,1]$ and right continuous at 0 ;

(3) $\underline{u}(r) \leq \bar{u}(r), 0 \leq r \leq 1$.

According to Zadeh's extension principle, the operation of addition on $\mathbb{E}$ (the space of all fuzzy numbers) is defined by

$$
(u+v)(x)=\sup _{y \in \mathbb{R}} \min \{u(y), v(x-y)\}, \quad x \in \mathbb{R},
$$

and scalar multiplication of a fuzzy number is given by

$$
(k \odot u)(x)= \begin{cases}u\left(\frac{x}{k}\right), & k>0, \\ \widetilde{0}, & k=0,\end{cases}
$$

where $\widetilde{0} \in \mathbb{E}$.

\section{Centroid Formula for Trapezoidal Fuzzy Number}

Let us consider $A$ to be a given trapezoidal fuzzy number such that $A=\left(a_{1}, a_{2}, a_{3}, a_{4}\right)$; then the centroid point of $A$ is obtained by Wang et al. [9] by

$$
\operatorname{COG}(A)=\left(\alpha_{A}, \beta_{A}\right),
$$

where

$$
\begin{gathered}
\alpha_{A}=\frac{1}{3}\left[a_{1}+a_{2}+a_{3}+a_{4}-\frac{a_{4} a_{3}-a_{1} a_{2}}{\left(a_{4}+a_{3}\right)-\left(a_{1}+a_{2}\right)}\right], \\
\beta_{A}=\frac{1}{3}\left[1+\frac{a_{3}-a_{2}}{\left(a_{4}+a_{3}\right)-\left(a_{1}+a_{2}\right)}\right] .
\end{gathered}
$$

Hence, for any triangular fuzzy number with a piecewise linear membership function, its centroid can be derived by

$$
\begin{gathered}
\alpha_{A}=\frac{a_{1}+a_{2}+a_{4}}{3}, \\
\beta_{A}=\frac{1}{3} .
\end{gathered}
$$

A briefly review about the relation between trapezoidal fuzzy number and its centroid formula is given.

Let us consider the left and right spreads, $\left(L_{A}, R_{A}\right)$, and COG point $\left(\alpha_{A}, \beta_{A}\right)$ of unknown fuzzy number $A$ is given. The goal is determining such unknown fuzzy number using mentioned information. To do this, we should solve the following linear system:

$$
\begin{gathered}
a_{2}-a_{1}=L_{A}, \\
a_{4}-a_{3}=R_{A}, \\
\alpha_{A}=\frac{1}{3}\left[a_{1}+a_{2}+a_{3}+a_{4}-\frac{a_{4} a_{3}-a_{1} a_{2}}{\left(a_{4}+a_{3}\right)-\left(a_{1}+a_{2}\right)}\right], \\
\beta_{A}=\frac{1}{3}\left[1+\frac{a_{3}-a_{2}}{\left(a_{4}+a_{3}\right)-\left(a_{1}+a_{2}\right)}\right] .
\end{gathered}
$$

However, in [7], the authors proved that system (7) is linear. To this end, Hadi-Vencheh and Allame [7] have obtained the elements $a_{1}, \ldots, a_{4}$ using the following formula explicitly:

$$
\begin{gathered}
a_{1}=\alpha_{A}+\frac{b_{0}^{2}-c_{0}^{2}-d_{0}^{2}-c_{0} d_{0}}{3\left(c_{0}+d_{0}-b_{0}\right)} \\
a_{2}=a_{1}+b_{0}, \\
a_{3}=a_{1}+c_{0}, \\
a_{4}=a_{1}+d_{0},
\end{gathered}
$$

where

$$
\begin{gathered}
b_{0}=L_{A}, \\
c_{0}=\frac{\left(3 \beta_{A}-1\right) R_{A}+\left(2-3 \beta_{A}\right) L_{A}}{3\left(1-2 \beta_{A}\right)}, \\
d_{0}=c_{0}+R_{A} .
\end{gathered}
$$

Now, we provide some results which will be useful to prove the triangular inequality for proposed distance measure (12). The proofs are so easy, so we omitted them.

Lemma 2. Let one consider the real values $a_{i}, i=1, \ldots, 6$ such that $a_{1} \leq a_{2}, a_{3} \leq a_{4}$, and $a_{5} \leq a_{6}$. Then, one has

$$
\max \left\{a_{1}, a_{3}, a_{5}\right\} \leq \max \left\{a_{2}, a_{4}, a_{6}\right\} .
$$


Lemma 3. Let one consider the real values $a_{i}, i=1, \ldots, 6$. Then, one has

$$
\begin{aligned}
\max & \left\{a_{1}+a_{2}, a_{3}+a_{4}, a_{5}+a_{6}\right\} \\
& \leq \max \left\{a_{1}, a_{3}, a_{5}\right\}+\max \left\{a_{2}, a_{4}, a_{6}\right\} .
\end{aligned}
$$

\section{Proposed Distance Measure for Trapezoidal Fuzzy Numbers}

In this section, we propose a new distance measure for trapezoidal fuzzy numbers using their centroids and left/right spread.

Definition 4. Let one consider two trapezoidal fuzzy numbers $A=\left(a_{1}, a_{2}, a_{3}, a_{4}\right)$ and $B=\left(b_{1}, b_{2}, b_{3}, b_{4}\right)$ with centroid points $\left(\alpha_{A}, \beta_{A}\right)$ and $\left(\alpha_{B}, \beta_{B}\right)$, left/right spreads $\left(L_{A}, R_{A}\right)$ and $\left(L_{B}, R_{B}\right)$, respectively. Then, the distance measure between two mentioned fuzzy numbers is as follows:

$$
d(A, B)=\max \left\{\left|\alpha_{A}-\alpha_{B}\right|,\left|\beta_{A}-\beta_{B}\right|,\left|L_{A}-L_{B}\right|,\left|R_{A}-R_{B}\right|\right\} .
$$

Now, we state the metric properties of $d$ proposed by (12).

Theorem 5. Let one consider distance measure (12) under the assumptions of Definition 4. Then, one has

$$
\begin{aligned}
& \text { (1) } d(A, B) \geq 0, \\
& \text { (2) } d(A, B)=d(B, A), \\
& \text { (3) } d(A, B)=0 \Longleftrightarrow A=B, \\
& \text { (4) } d(A, C) \leq d(A, B)+d(B, C),
\end{aligned}
$$

for all arbitrary trapezoidal fuzzy numbers $A, B$, and $C$.

Proof. The proofs of the first and the second properties are an easy work, so we omitted them. Now, we prove the third property. Let us consider $A=B$; then we deduce that $\alpha_{A}=\alpha_{B}$, $\beta_{A}=\beta_{B}$, and $L_{A}=L_{B}, R_{A}=R_{B}$, and then using (12) we obtain $d(A, B)=0$. Conversely, Let us consider $d(A, B)=0$; then using (12) we have

$$
\max \left\{\left|\alpha_{A}-\alpha_{B}\right|,\left|\beta_{A}-\beta_{B}\right|,\left|L_{A}-L_{B}\right|,\left|R_{A}-R_{A}\right|\right\}=0,
$$

which leads to obtain

$$
\begin{aligned}
& \left|\alpha_{A}-\alpha_{B}\right|=0, \\
& \left|\beta_{A}-\beta_{B}\right|=0, \\
& \left|L_{A}-L_{B}\right|=0, \\
& \left|R_{A}-R_{B}\right|=0 .
\end{aligned}
$$

Hence, we have

$$
\alpha_{A}=\alpha_{B}, \quad \beta_{A}=\beta_{B}, \quad L_{A}=L_{B}, \quad R_{A}=R_{B} .
$$

Using obtained result (16), we prove that $a_{i}=b_{i}, i=1,2,3,4$ which completes the proof of this part.
Now, we use the following notations:

$$
b_{2}=b_{1}+f_{0}, \quad b_{3}=b_{1}+g_{0}, \quad b_{4}=b_{1}+h_{0} .
$$

Similar to the results obtained for $a_{1}, \ldots, a_{4}$, we have $f_{0}=L_{B}$, $h_{0}=g_{0}+R_{B}$, and

$$
g_{0}=\frac{\left(3 \beta_{B}-1\right) R_{B}+\left(2-3 \beta_{B}\right) L_{B}}{3\left(1-2 \beta_{B}\right)} .
$$

Obviously, using (16), we deduce that $g_{0}=c_{0}$. Moreover, since $d_{0}=c_{0}+R_{A}$ and $h_{0}=g_{0}+R_{B}$; we derive $d_{0}=h_{0}$. Also, $b_{0}=L_{A}$ and $f_{0}=L_{B}$, using (16) we obtain $b_{0}=f_{0}$. So, using obtained results we have

$$
\frac{b_{0}^{2}-c_{0}^{2}-d_{0}^{2}-c_{0} d_{0}}{3\left(c_{0}+d_{0}-b_{0}\right)}=\frac{f_{0}^{2}-g_{0}^{2}-h_{0}^{2}-g_{0} h_{0}}{3\left(g_{0}+h_{0}-f_{0}\right)} .
$$

Using

$$
\begin{aligned}
& a_{1}=\alpha_{A}+\frac{b_{0}^{2}-c_{0}^{2}-d_{0}^{2}-c_{0} d_{0}}{3\left(c_{0}+d_{0}-b_{0}\right)}, \\
& b_{1}=\alpha_{B}+\frac{f_{0}^{2}-g_{0}^{2}-h_{0}^{2}-g_{0} h_{0}}{3\left(g_{0}+h_{0}-f_{0}\right)},
\end{aligned}
$$

and (16), we deduce that $a_{1}=b_{1}$.

Now, we demonstrate that $a_{i}=b_{i}, i=2,3,4$. Since, $a_{2}=$ $a_{1}+L_{A}$ and $b_{2}=b_{1}+L_{B}$. Using the fact that $L_{A}=L_{B}$ and $a_{1}=b_{1}$, we deduce that $a_{2}=b_{2}$. Moreover, $a_{3}=a_{1}+c_{0}$ and $b_{3}=b_{1}+g_{0}$. Then, using the fact that $c_{0}=g_{0}$ and $a_{1}=b_{1}$ we obtain $a_{3}=b_{3}$. Finally, since $d_{0}=h_{0}$, we derive that $a_{4}=$ $a_{1}+d_{0}$ and $b_{4}=b_{1}+h_{0}$ are equal. The proof of this part is completed.

Now, we prove the triangular inequality for the proposed distance measure (12). Let us consider three trapezoidal fuzzy numbers $A=\left(a_{1}, a_{2}, a_{3}, a_{4}\right), B=\left(b_{1}, b_{2}, b_{3}, b_{4}\right)$, and $C=$ $\left(c_{1}, c_{2}, c_{3}, c_{4}\right)$. Then, using Lemmas 2 and 3 and (12), we have

$$
\begin{aligned}
d(A, C) & \\
= & \max \left\{\left|\alpha_{A}-\alpha_{C}\right|,\left|\beta_{A}-\beta_{C}\right|,\left|L_{A}-L_{C}\right|,\left|R_{A}-R_{C}\right|\right\} \\
\leq & \max \left\{\left|\alpha_{A}-\alpha_{B}\right|+\left|\alpha_{B}-\alpha_{C}\right|,\left|\beta_{A}-\beta_{B}\right|+\left|\beta_{B}-\beta_{C}\right|,\right. \\
& \left.\left|L_{A}-L_{B}\right|+\left|L_{B}-L_{C}\right|,\left|R_{A}-R_{B}\right|+\left|R_{B}-R_{C}\right|\right\} \\
\leq & \max \left\{\left|\alpha_{A}-\alpha_{B}\right|,\left|\beta_{A}-\beta_{B}\right|,\left|L_{A}-L_{B}\right|,\left|R_{A}-R_{B}\right|\right\} \\
& +\max \left\{\left|\alpha_{B}-\alpha_{C}\right|,\left|\beta_{B}-\beta_{C}\right|,\left|L_{B}-L_{C}\right|,\left|R_{B}-R_{C}\right|\right\} \\
= & d(A, B)+d(B, C),
\end{aligned}
$$

which completes the proof.

Remark 6. Indeed, we show that if the difference between centroids and also the difference between left spreads and right spreads of two given trapezoidal fuzzy numbers go to zero, we can conclude that the mentioned fuzzy numbers are identical. To the best of our knowledge, this is the first time in 
the literature that each fuzzy number is characterized using its centroid formula and its left/right spread uniquely. Also, our discussion completes Hadi-Vencheh and Allame's paper [7] about the relation between fuzzy number and its centroid formula.

\section{Conclusion}

Recently, Hadi-Vencheh and Allame [7] have proposed the relation between trapezoidal fuzzy number and its centroid formula. To do so, they proved that one can easily obtain such relation by solving linear system (7). However, they did not consider the uniqueness of solution. In other words, if the centroids and left/right spreads of two trapezoidal fuzzy numbers are equal, how can we ensure that these fuzzy numbers are identical? This paper answered this question.

So, we proposed a new distance measure based on the differences between centroids and left/right spreads. Consequently, we proved the metric properties of it. To the best of our knowledge, this is the first time in the literature that such construction is applied. Indeed, the uniqueness of solution in this area is so important which has not been investigated yet.

For future research, we will apply (12) to obtain some new similarity measures and ranking fuzzy numbers [10-16] and risk analysis [17] and also will use it to determine the nearest approximation of fuzzy numbers [18].

\section{References}

[1] L. A. Zadeh, "Fuzzy sets," Information and Computation, vol. 8, pp. 338-353, 1965.

[2] A. Kaufmann and M. M. Gupta, Introduction to Fuzzy Arithmetic: Theory and Application, Van Nostrand Reinhold, New York, NY, USA, 1991.

[3] H. J. Zimmermann, Fuzzy sets theory and its applications, Kluwer Academic, Dordrecht, The Netherlands, Second edition, 1992.

[4] A. Ban, L. Coroianu, and P. Grzegorzewski, "Trapezoidal approximation and aggregation," Fuzzy Sets and Systems, vol. 177, pp. 45-59, 2011.

[5] P. Grzegorzewski, "Metrics and orders in space of fuzzy numbers," Fuzzy Sets and Systems, vol. 97, no. 1, pp. 83-94, 1998.

[6] P. Grzegorzewski, "Distances between intuitionistic fuzzy sets and/or interval-valued fuzzy sets based on the Hausdorff metric," Fuzzy Sets and Systems, vol. 148, no. 2, pp. 319-328, 2004.

[7] A. Hadi-Vencheh and M. Allame, "On the relation between a fuzzy number and its centroid," Computers \& Mathematics with Applications, vol. 59, no. 11, pp. 3578-3582, 2010.

[8] M. Friedman, M. Ma, and A. Kandel, "Numerical solutions of fuzzy differential and integral equations," Fuzzy Sets and Systems, vol. 106, no. 1, pp. 35-48, 1999, Fuzzy modeling and dynamics.

[9] Y. M. Wang, J. B. Yang, D.-L. Xu, and K.-S. Chin, "On the centroids of fuzzy numbers," Fuzzy Sets and Systems, vol. 157, no. 7, pp. 919-926, 2006.

[10] S. Abbasbandy and B. Asady, "Ranking of fuzzy numbers by sign distance," Information Sciences, vol. 176, no. 16, pp. 2405-2416, 2006.
[11] S. Abbasbandy and T. Hajjari, "A new approach for ranking of trapezoidal fuzzy numbers," Computers \& Mathematics with Applications, vol. 57, no. 3, pp. 413-419, 2009.

[12] B. Asady, "The revised method of ranking LR fuzzy number based on deviation degree," Expert Systems with Applications, vol. 37, no. 7, pp. 5056-5060, 2010.

[13] B. Asady and A. Zendehnam, "Ranking fuzzy numbers by distance minimization," Applied Mathematical Modelling, vol. 31, no. 11, pp. 2589-2598, 2007.

[14] S. Salahshour, S. Abbasbandy, and T. Allahviranloo, "Ranking fuzzy numbers using fuzzy maximizingminimizing points," in Proceedings of the 7th Conference of the European Society for Fuzzy Logic and Technology (IFSA-EUSFLAT '11), vol. 1 of Advances in Intelligent Systems Research, pp. 763-769, 2011.

[15] Z. X. Wang, Y. J. Liu, Z. P. Fan, and B. Feng, "Ranking L-R fuzzy number based on deviation degree," Information Sciences, vol. 179, no. 13, pp. 2070-2077, 2009.

[16] Y. M. Wang and Y. Luo, "Area ranking of fuzzy numbers based on positive and negative ideal points," Computers \& Mathematics with Applications, vol. 58, no. 9, pp. 1769-1779, 2009.

[17] S. M. Chen and J. H. Chen, "Fuzzy risk analysis based on ranking generalized fuzzy numbers with different heights and different spreads," Expert Systems with Applications, vol. 36, no. 3, pp. 6833-6842, 2009.

[18] P. Grzegorzewski, "Nearest interval approximation of a fuzzy number," Fuzzy Sets and Systems, vol. 130, no. 3, pp. 321-330, 2002. 


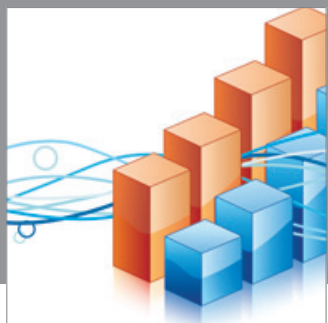

Advances in

Operations Research

mansans

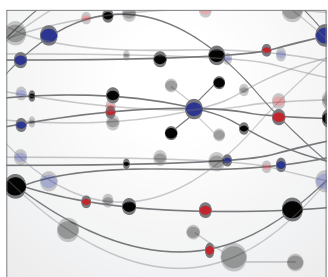

The Scientific World Journal
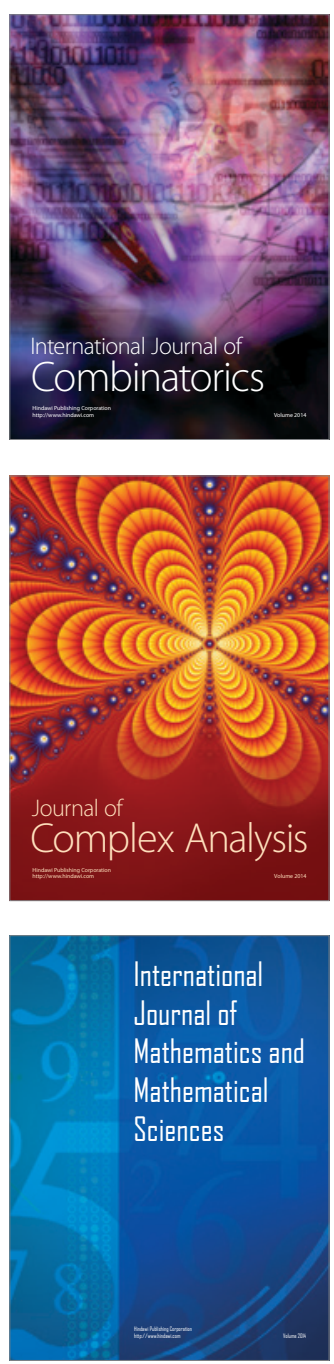
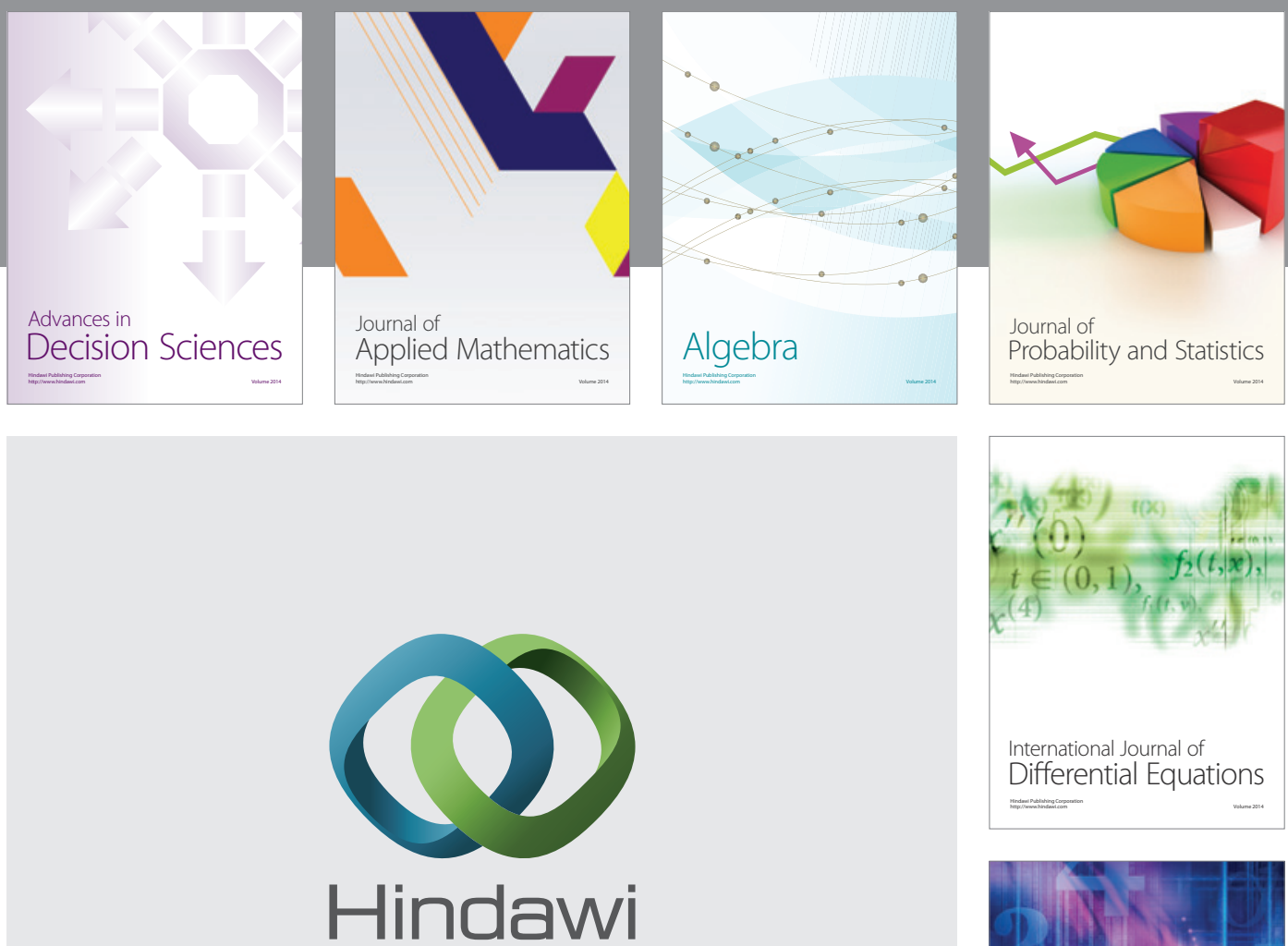

Submit your manuscripts at http://www.hindawi.com
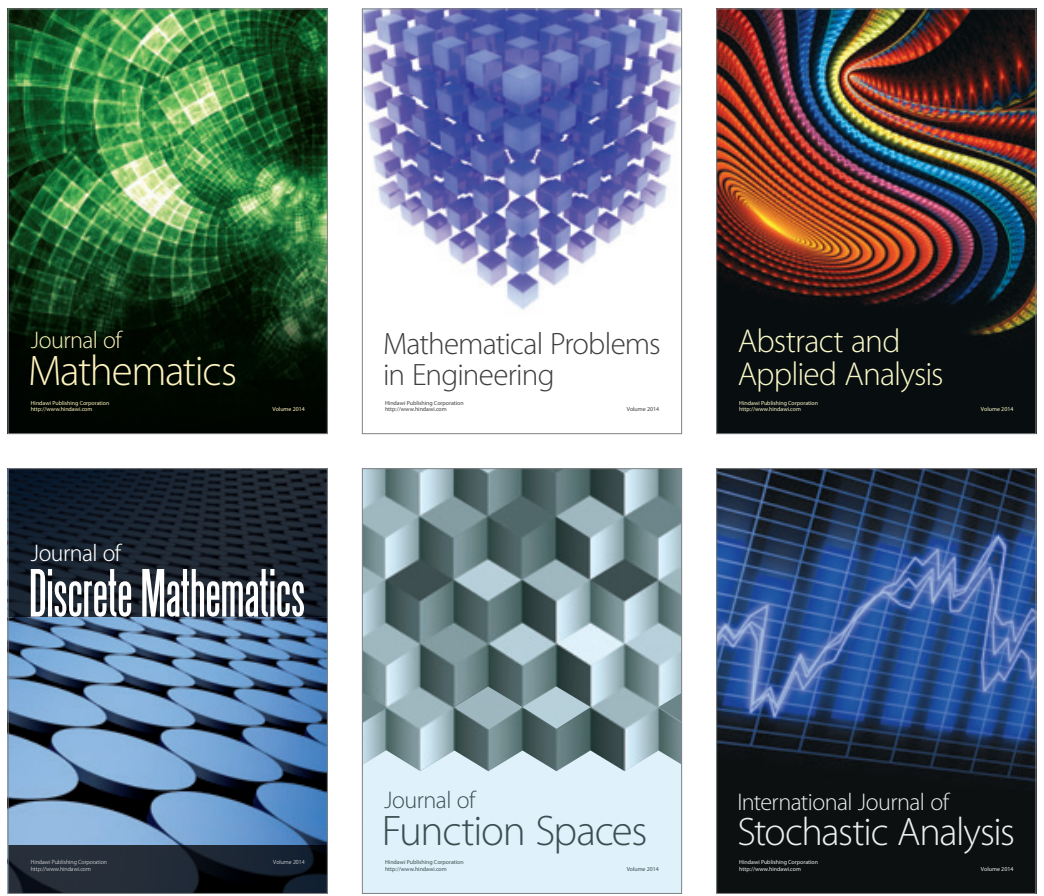

Journal of

Function Spaces

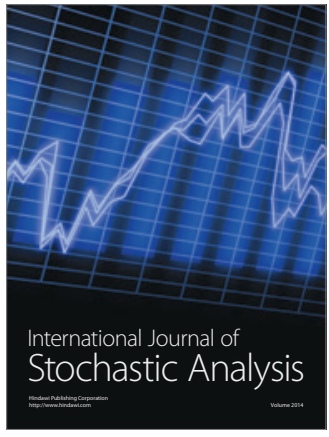

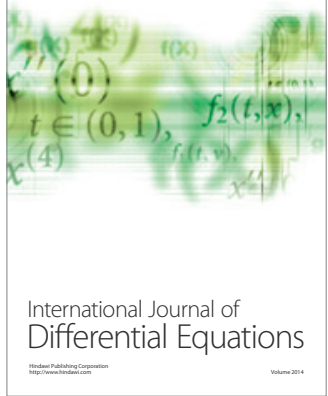
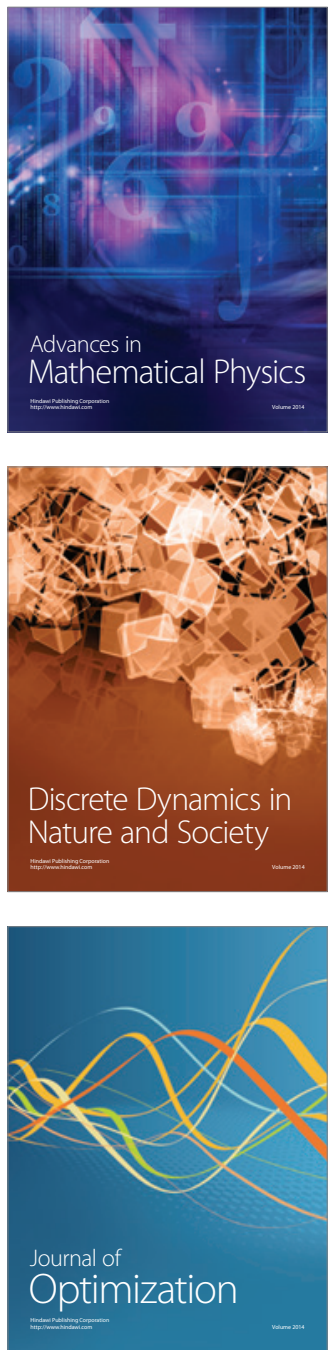